Ensino, Saúde e Ambiente - V 7 (1), Edição Especial, maio de 2014

\title{
ANÁLISE DA QUALIDADE DE VIDA DO PROFESSOR DA ÁREA DE SAÚDE NO ENSINO SUPERIOR NO SUL DO ESTADO DO ESPÍRITO SANTO
}

\section{LIFE QUALITY ANALYSIS OF HEALTH SECTOR PROFESSOR ON COLLEGE EDUCATION IN THE SOUTH OF ESPÍRITO SANTO STATE}

\author{
Nelson Coimbra Ribeiro Neto ${ }^{1}$ \\ Fabiana Abrahão ${ }^{2}$ \\ ${ }^{1}$ Centro Universitário São Camilo - Espírito Santo / Curso de Fisioterapia / coimbra.fisio @ gmail.com \\ ${ }^{2}$ Centro Universitário Anhanguera de Niterói - UNIAN / Programa de Mestrado Profissional em Ciências \\ da Saúde e do Ambiente / fabiana.abrahao@yahoo.com.br
}

\section{RESUMO}

Com o objetivo de analisar a qualidade de vida do professor na área de saúde, no Ensino Superior do sul do Espírito Santo, indagamos se o profissional da saúde teria uma melhor qualidade de vida do que professores de outras áreas. Esta foi uma pesquisa quantitativa, com observação participativa estruturada, aplicando-se o questionário SF 36 a 50 docentes, onde 25 eram de cursos da área da Saúde, e outros 25 compunham o grupo Controle. Na análise estatística utilizou-se os testes de Shapiro-Wilk, t de student e de Mann-Whitney $U$, além das correlações de Pearson e Spearman. Apenas os domínios (Dor) e (Estado Geral de Saúde) apresentaram diferença significativa, e observamos correlação entre idade e tempo de atuação como professor no Ensino Superior. Para atingir estes professores e transformá-los em multiplicadores dos cuidados em saúde sugerimos a implementação de programas de qualidade de vida nas Instituições.

Palavras-chave: saúde do trabalhador; qualidade de vida; docente; ensino superior.

\begin{abstract}
Within the aim of analyzing the life quality of health sector professor on Graduation in the south of Espirito Santo, we ask if the health professional would have a better life quality than teachers in other areas. This was a quantitative survey through a participant structured observation, applying the SF-36 questionnaire to 50 teachers, which 25 teachers were from Health area courses, and other 25 teachers formed a control group. In the statistical analysis we used the Shapiro-Wilk test, Student's $t$ and Mann-Whitney U, besides the Pearson and Spearman correlations. Only domains (pain) and (General Health) showed significant differences and correlations between age and time working as a teacher in Graduation. To achieve these teachers and transforming them into multipliers in health care, we suggest the implementation of life quality programs on these Institutions.
\end{abstract}

Keywords: worker health; life quality; professor; college education.

\section{Introdução}

$\mathrm{O}$ ambiente de ensino onde se insere o professor geralmente o leva a uma sobrecarga em suas funções, podendo promover determinado grau de insatisfação, de 
difícil mensuração devido à subjetividade inerente. Este prisma pode não estimular o docente a cuidar de sua saúde, como trabalhador que é. Sob esta perspectiva, perguntamos se as dificuldades envolvem a escassez de tempo e outras condições favoráveis para fazê-lo, como observado por Soares et al (2011). Os docentes, pelo seu nível de instrução e acesso aos mais diversos serviços de saúde compreendem as variáveis de sua qualidade de vida, seja laboral ou familiar, porém, devido às condições de trabalho impostas nem sempre as praticam. Eles "devem estimular o cuidado de si na graduação, colocando-o em prática, para que os futuros (...) professores (...) tenham a consciência de que também são profissionais e precisam ser cuidados; necessitam olhar para si e cuidar de si" (SOARES et al, 2011).

Tani (2002) trata do tema qualidade de vida de uma forma muito mais ampla: “(...) ter saúde física e mental, relações sociais harmoniosas e construtivas, educação permanente, relacionamento respeitoso e amigável com o meio ambiente, tempo livre para o lazer e oportunidades para usufruir a cultura em sua plenitude”. Percebendo que é possível propiciar aos professores de uma Instituição de Ensino Superior uma melhora significativa em sua qualidade de vida, esta pesquisa se insere por meio de um produto a ser implementado aos projetos e programas de qualidade de vida já em andamento em uma Instituição de Ensino Superior (IES) do sul do Estado do Espírito Santo, atingindo o objetivo de um Programa de Mestrado Profissional, por meio da promoção da transformação do meio social e laboral do mestrando.

Direcionando-nos aos professores, obervamos uma rotina capitalista cercada por tarefas, prazos e metas, onde para obter sucesso profissional e familiar, por meio da produção e consumo, é permitido se olvidar da própria saúde. O contexto é que este período de esquecimento geralmente não é curto, durando muitas vezes uma ou mais décadas, quando seu organismo já não responde tão rapidamente às mudanças de estilos de vida.

Tomemos agora um recorte desta realidade apresentada, não como verdade absoluta, e sim como possibilidade concreta de estilos de vida levados por diversos profissionais do mundo atual, e observemos os docentes oriundos de cursos da área de Saúde. Estes por sua própria formação tecnicista e não licenciada já se diferem em demasia dos letrados, e provavelmente consomem maior tempo para suas funções de ensino-aprendizagem, o que talvez provoque ainda mais a possibilidade da escassez temporal para seu autocuidado em saúde aqui sugerida. A princípio é permitido imaginar que um profissional de saúde tende a conhecer melhor seu organismo, tanto Edição Especial com os melhores trabalhos apresentados no IV ENECiências: UFF - 13 a 16 de maio de 2014. 
fisiologicamente quanto filosoficamente, o que nos induz ao raciocínio de que um professor universitário, profissional de saúde, tende a cuidar melhor de si próprio e de promover o cuidado em saúde para seus pupilos e sociedade em geral.

\section{Material e métodos}

Esta foi uma pesquisa quantitativa, de caráter exploratório, descritivo e indutivo, por meio de observação participativa estruturada, uma vez que o pesquisador principal é parte do universo de colaboradores analisados por meio de uma amostra. A característica quantitativa se dá pela aplicação do questionário Medical Outcomes Study Questionaire 36 - Item Short Form Health Survey (SF-36) e análise estatística dos resultados obtidos pelas amostras, obtidas entre junho e julho de 2013. Dos atuais 165 docentes do Centro Universitário São Camilo - Espírito Santo, 50 professores da graduação foram entrevistados $(30,30 \%)$, sendo 25 docentes de 5 cursos da área da Saúde, e um grupo controle também de 25 professores de outros cursos de graduação não relacionados à área de Saúde dessa mesma Instituição de Ensino Superior, onde não houve perda amostral ao longo do desenvolvimento da pesquisa. Os professores do grupo "Saúde" são oriundos dos cursos de Educação Física, Enfermagem, Farmácia, Fisioterapia e Nutrição, devido às afinidades sob a ótica das Diretrizes Curriculares Nacionais pertinentes (FRAUCHES, 2008). Já a amostra "Controle” foi obtida por meio dos cursos de Administração, Direito, História, Pedagogia e Sistemas de Informação, como preconiza Lakatos \& Marconi (2011), sobre grupos controle.

Como fatores de inclusão para conduzir a escolha da amostra, os voluntários possuíam, à época da coleta de dados, vínculo empregatício vigente com a referida IES em seu nível de ensino superior por pelo menos um ano, independente de experiência docente anterior, idade, gênero ou etnia. Foram excluídos da amostra aqueles que também possuíam vínculo administrativo paralelo ao acadêmico, além dos que viessem a ter seus contratos profissionais rescindidos ao longo da pesquisa, o que não ocorreu com nenhum dos participantes. Todos estes fatores de inclusão e exclusão foram repassados aos respectivos Coordenadores de Cursos envolvidos para que os mesmos selecionassem seus 5 (cinco) docentes. Observamos que os Coordenadores entregaram aos participantes o questionário de qualidade de vida e um termo de consentimento livre e esclarecido, fornecidos pelo pesquisador, antes de suas entrevistas. Foi resguardado o 
direito em desistência, mesmo que sem justificativa, desde que houvesse comunicação formalizada por escrito ao pesquisador principal.

Esta pesquisa não trouxe nenhum tipo de risco à saúde dos voluntários envolvidos, exceto o aspecto emocional, pois o preenchimento dos questionários foi solicitado pelo respectivo coordenador de curso. Ao mesmo tempo pode beneficiá-los por meio do estímulo em cuidar melhor de sua própria saúde, em seu ambiente laboral, transformando-o em um agente multiplicador mais ativo dos cuidados em saúde. Com base na Resolução 466/12 do Conselho Nacional de Saúde do Brasil, esta pesquisa foi autorizada pelo parecer consubstanciado no 314.631 do Comitê de Ética e Pesquisa da União Social Camiliana (Centro Universitário São Camilo - UNISC).

A coleta de dados para a pesquisa foi realizada por meio do questionário genérico semiaberto SF-36, validado internacionalmente e traduzido no Brasil para a língua portuguesa por Ciconelli et al (1999); organizado pelo pesquisador principal, e garantindo a privacidade e confidência das informações, uma vez que os questionários foram entregues lacrados aos pesquisados. Todos os respondentes dispuseram de um curto espaço de tempo para responderem ao questionário, não sendo permitido levá-lo para seus domicílios. As pontuações obtidas pelo SF-36 e os cálculos de Raw Scales respeitam sua tabulação validada internacionalmente, mediante cálculo específico dos escores para cada uma das 11 (onze) questões existentes.

Posteriormente foi realizada análise estatística dos dados coletados a fim de subsidiar o desenvolvimento da "Cartilha da Saúde Docente", produto de dissertação de Mestrado Profissional. Utilizou-se o teste de Shapiro-Wilk para verificar a normalidade dos domínios obtidos pelo questionário SF-36, onde 4 domínios $(1,2,6$ e 7) se mostraram não paramétricos e outros 4 domínios $(3,4,5$ e 8$)$ se apresentaram dento da curva de Gauss. Posteriormente foi aplicado o teste $t$ de student não pareado para os domínios paramétricos e o teste de Mann-Whitney $U$ para os domínios não paramétricos. Para correlações entre o tempo de profissão e a idade, utilizou-se o teste de correlação de Pearson para o grupo Saúde, que se apresentou dentro da normalidade por meio do teste de Shapiro-Wilk, e o teste de correlação de Spearman para o grupo Controle, que não passou pelo teste de Shapiro-Wilk. Todos os testes estatísticos utilizaram o software GraphPad Prism 5 para o sistema operacional Windows ${ }^{\circledR}$. 


\section{Resultados e discussão}

Os dados coletados $(\mathrm{n}=50)$ por meio do questionário multidimensional SF-36, composto por 36 itens, que segundo Grincenkov, et al (2011) é caracterizado pela autoavaliação do paciente, o que nos permite aferir possibilidades de influência subjetiva em seu preenchimento; foram alocados em dois grupos distintos: um denominado "Saúde" e outro "Controle". Cada um dos grupos contou com 25 (vinte e cinco) participantes de 5 (cinco) cursos de graduação distintos por grupo, onde é possível a análise comparativa entre grupos e entre cursos.

A princípio, obtivemos os dados de distribuição por gênero, idade e tempo de atuação como professor de nível superior dos participantes, onde se identificou que o grupo Saúde possuía $76 \%$ de mulheres $(n=19)$ e $24 \%$ de homens $(n=6)$. Já o grupo controle possuía $60 \%$ de mulheres $(n=15)$ e $40 \%$ de homens. $(n=10)$. Isto demonstra claramente uma superioridade do gênero feminino em ambos os grupos, em especial no Saúde. Totalizando, $68 \%$ da amostra $(n=50)$ eram mulheres, e $32 \%$ homens.

Quanto à idade e ao tempo de atuação profissional no ensino superior foi possível observar que os docentes da área de saúde demoram um pouco mais para iniciar no Ensino Superior do que os professores formados por cursos de outras áreas. Assim, observamos pela tabela 1 que a média de idade para o grupo Saúde (33,92 anos $\pm 6,05)$ foi similar à do grupo Controle $(35,96 \pm 6,57)$. E que a média de tempo de profissão no ensino superior foi maior para o grupo Controle $(9,16 \pm 5,72)$ do que para o grupo Saúde $(5,82 \pm 3,38)$.

Tabela 1: dados de gênero, idade \pm DP e tempo de atuação \pm DP.

CARACTERIZAÇÃO DA AMOSTRA

\begin{tabular}{|c|c|c|c|c|c|c|c|}
\hline \multirow{2}{*}{ GRUPOS } & \multirow{2}{*}{ AMOSTRA } & \multicolumn{2}{|c|}{ GÊNERO } & \multicolumn{2}{|l|}{ IDADE } & \multicolumn{2}{|c|}{ TEMPO DE PROFISSÃO } \\
\hline & & $\mathrm{M}$ & $\mathrm{F}$ & EM ANOS & $\mathrm{DP}$ & EM ANOS & DP \\
\hline Saúde & 25 & 6 & 19 & 33,92 & 6,05 & 5,82 & 3,38 \\
\hline Controle & 25 & 10 & 15 & 35,96 & 6,57 & 9,16 & 5,72 \\
\hline
\end{tabular}

Fonte: dados coletados e tabulados pelos autores

Edição Especial com os melhores trabalhos apresentados no IV ENECiências: UFF - 13 a 16 de maio de 2014. 
Na figura 1 é possível traçar um paralelo visual entre idade e tempo de atuação no Ensino Superior. Chamamos a atenção para uma comparação entre os dois cursos que apresentaram as maiores médias de idade entre seus cinco professores: Enfermagem e Pedagogia. Isso nos leva à compreensão que o profissional de Enfermagem que atua na docência em Instituição de Ensino Superior demora muito mais para iniciar suas atividades como professor; o que parece ser um padrão encontrado nesta pesquisa quando se compara os grupos Saúde e Controle.

Figura 1: média de idade e de tempo de atuação no Ensino Superior.

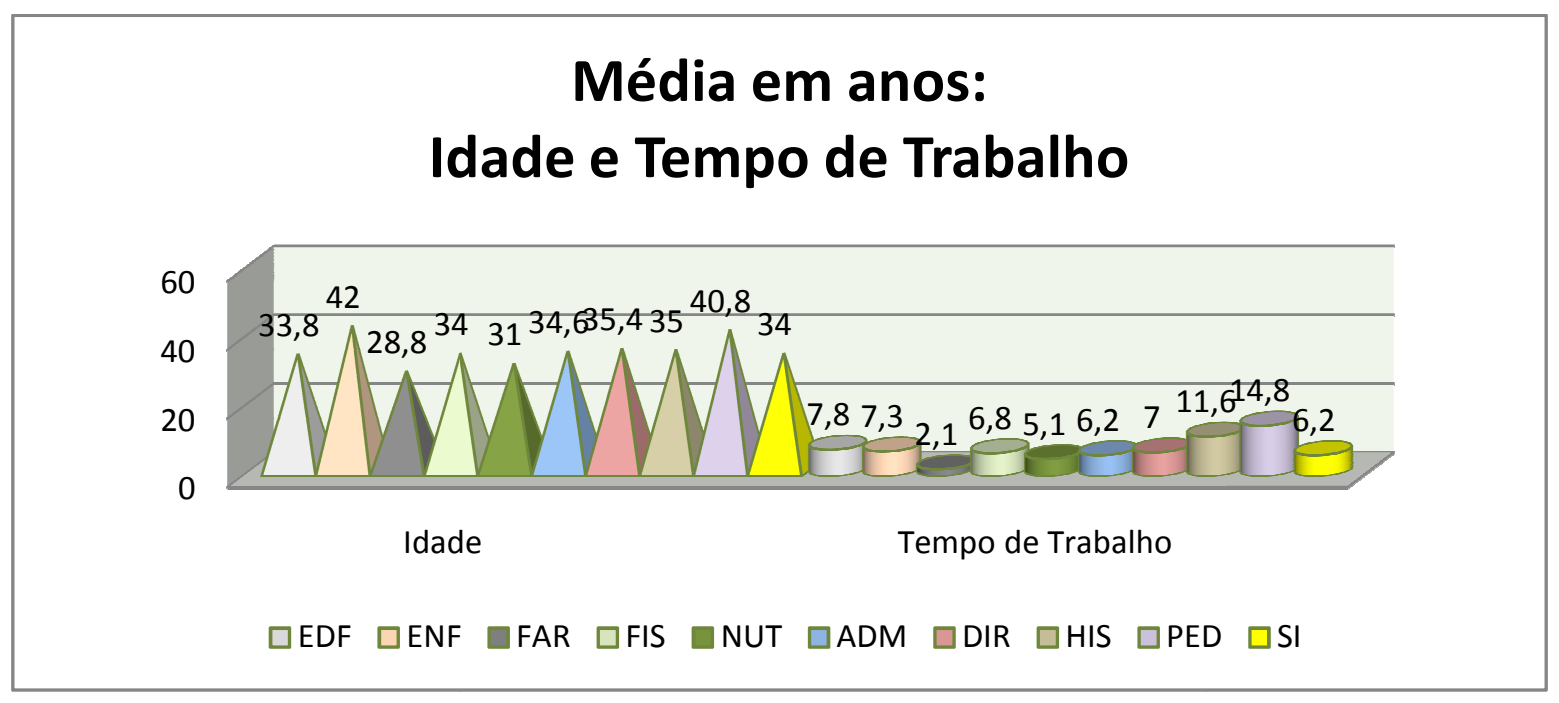

Fonte: dados coletados e tabulados pelos autores

Como demonstrado pelas figuras 2 e 3 , foi traçada uma correlação entre o tempo de profissão e a idade das amostras do grupo Saúde e Controle, considerando-se um valor de $\mathrm{p}=0,0102$ para Saúde, por meio do teste de correlação de Pearson, e de p=0,0475 para o grupo Controle, por meio do teste de correlação de Spearman. Em ambas as correlações adotou-se valor de p<0,05. Estudos de Souza \& Costa (2011) sobre a qualidade de vida de uma amostra de profissionais de educação física aferiram que o tempo de atuação profissional foi significativo em algumas dimensões (domínios) do questionário SF-36, onde quanto maior o tempo de trabalho, pior seria o indicador de qualidade de vida. Em tempo, muitos estudos apontam uma relação inversamente proporcional entre a carga horária da jornada de trabalho e uma pior qualidade de vida e incidência de processos patológicos; apesar de Souza \& Costa (2011) não terem encontrado diferença estatisticamente significativa entre carga horária e qualidade de vida em seus estudos. 
Figura 2: correlação entre tempo de profissão no Ensino Superior e idade para o grupo Saúde.

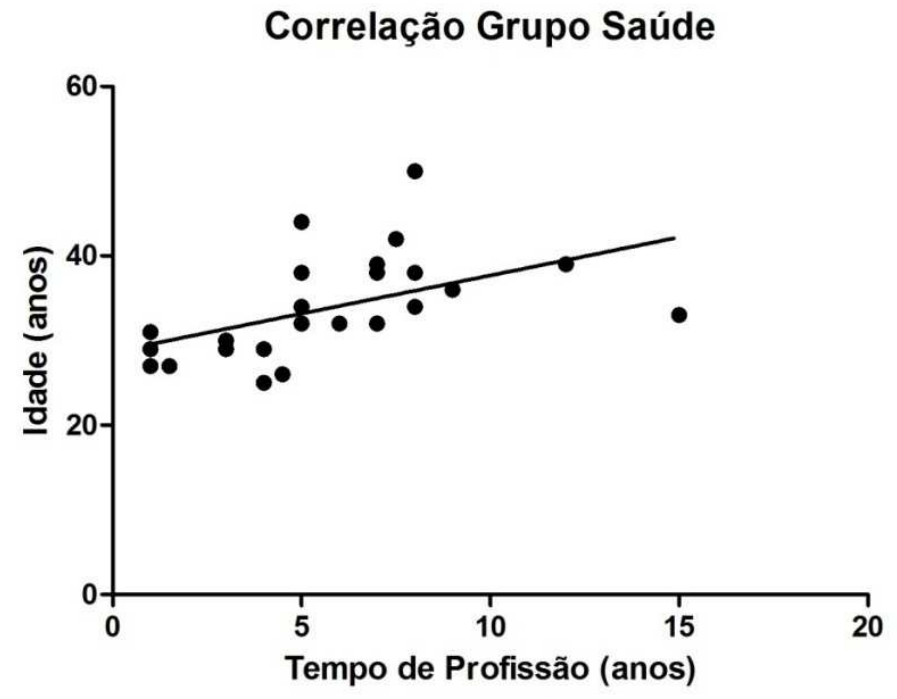

Fonte: dados coletados e tabulados pelos autores

Figura 3: correlação entre tempo de profissão no Ensino Superior e idade para o grupo Controle.

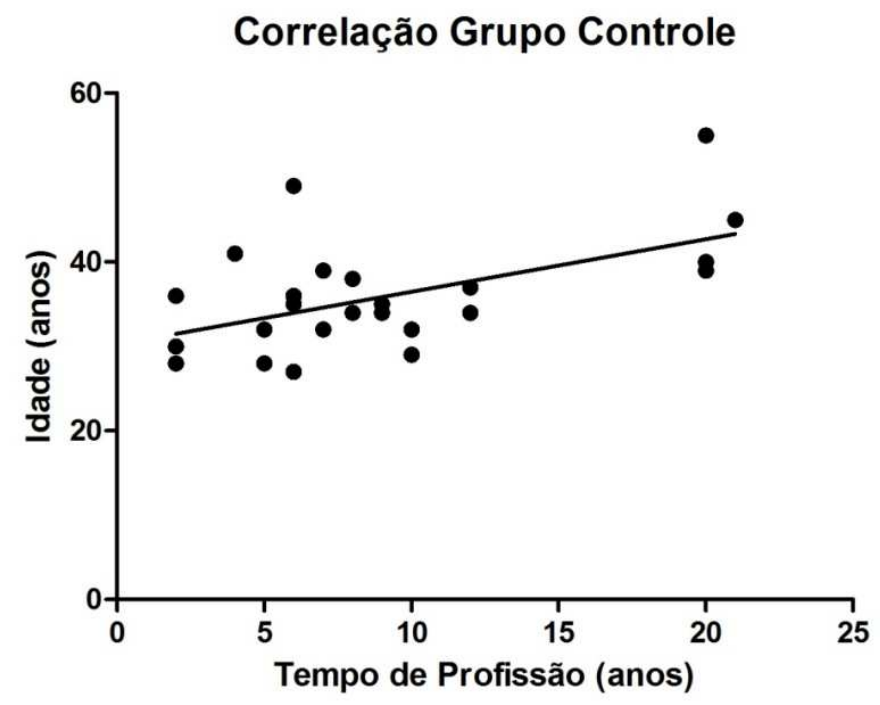

Fonte: dados coletados e tabulados pelos autores

De acordo com os critérios de análise do questionário SF-36 expostos na metodologia desta dissertação, todos os cálculos das onze pontuações e todos os raw scales para os oito domínios de cada participante foram devidamente obtidos de acordo com o preconizado pelo instrumento. Galvão, et al. (2007) analisaram que a qualidade de vida no climatério aumenta proporcionalmente à faixa etária, especialmente acima dos 60 anos. Já em nossa pesquisa pode-se notar que apesar da maior parte da amostra ser do gênero feminino (68\%), apresenta-se uma média etária geral baixa (34,94 anos).

Edição Especial com os melhores trabalhos apresentados no IV ENECiências: UFF - 13 a 16 de maio de 2014. 
Desta informação pode-se aferir que para um grupo de docentes em uma faixa etária mais elevada os resultados poderiam ser estatisticamente distintos.

A seguir nas figuras 4 e 5 , as médias com desvio padrão para os domínios obtidos nos grupos Saúde e Controle, onde os domínios 1 (Capacidade funcional), 2 (Limitação por aspectos físicos), 5 (Vitalidade), 6 (Aspectos sociais), 7 (Limitação por aspectos emocionais) e 8 (Saúde mental) foram estatisticamente semelhantes entre os dois grupos, o que segundo a pesquisa de Santos, et al. (2008) pode-se afirmar que os grupos eram comparáveis.

Após verificar os dados de Raw Scale de cada grupo, contatou-se que alguns domínios não se apresentavam dentro da normalidade por meio do teste de ShapiroWilk. Assim, para comparar um determinado domínio entre os dois grupos foi necessário primeiro verificar quais domínios seriam paramétricos e quais eram não paramétricos. Para os que se apresentavam dentro da curva de Gauss (3, 4, 5 e 8) adotou-se o teste $t$ de student não pareado, e para os demais (1, 2, 6 e 7) o teste de Mann-Whitney $U$ foi adotado.

Figura 4: médias + DP dos domínios obtidos no grupo Saúde.

\section{Domínios - Grupo Saúde}

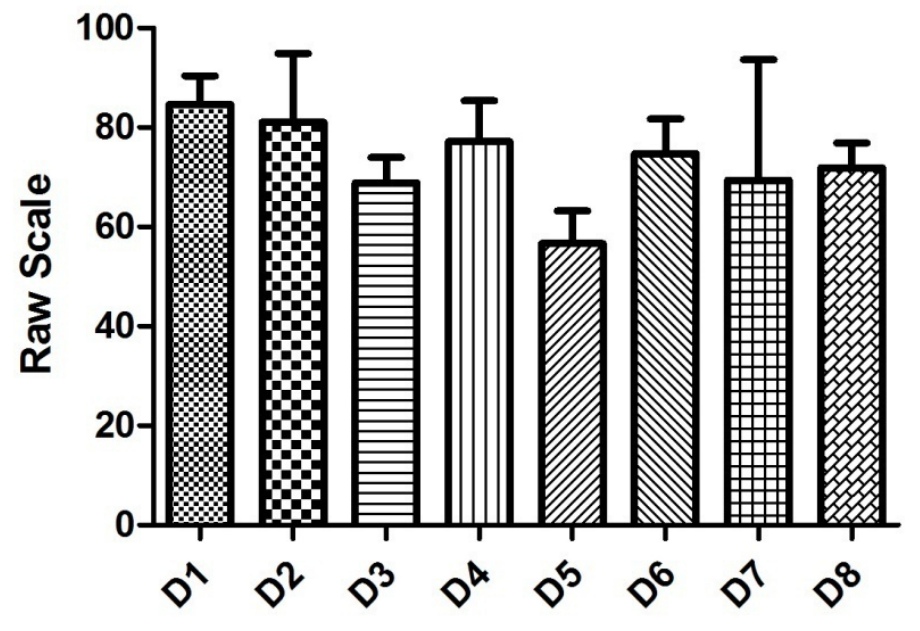

Fonte: dados coletados e tabulados pelos autores 
Figura 5: médias + DP dos domínios obtidos no grupo Controle.

Domínios - Grupo Controle

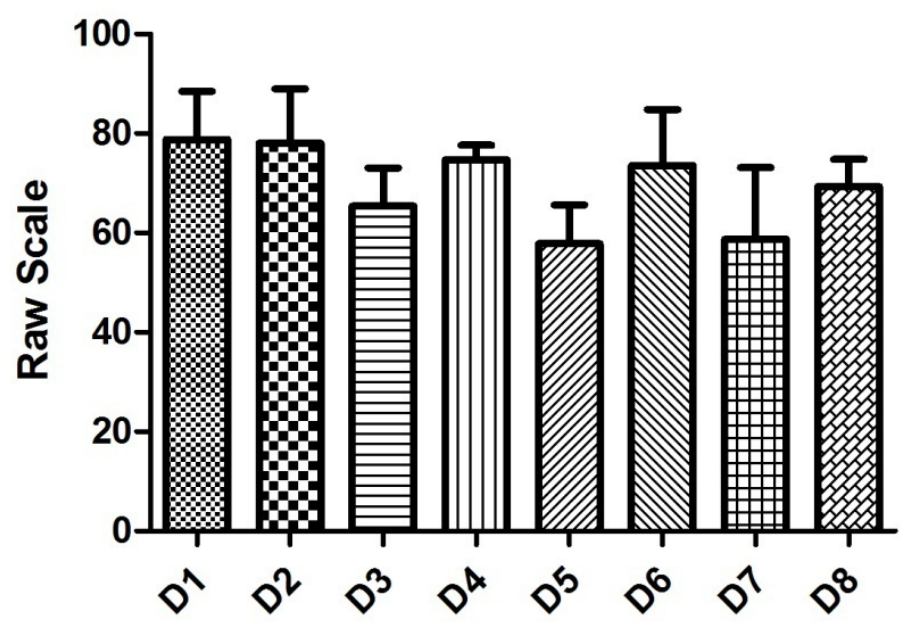

Fonte: dados coletados e tabulados pelos autores

Estatisticamente observamos que apenas os domínios 3 (Dor) e 4 (Estado geral de saúde - EGS) apresentaram diferença entre os grupos, para um valor de significância de $\mathrm{p}<0,05$, como apontado pelas figuras 6 e 7 abaixo. De acordo com Mota; Laurindo; Santos Neto (2010) é possível atribuir diferenças apresentadas pelos domínios à subjetividade das respostas ao questionário utilizado.

Figura 6: comparação do domínio 3 (dor) entre os grupos Saúde e Controle, por meio do teste t de student não pareado $(\mathbf{p}=\mathbf{0 , 0 0 9 6})$.

\section{Dor (DOR)}

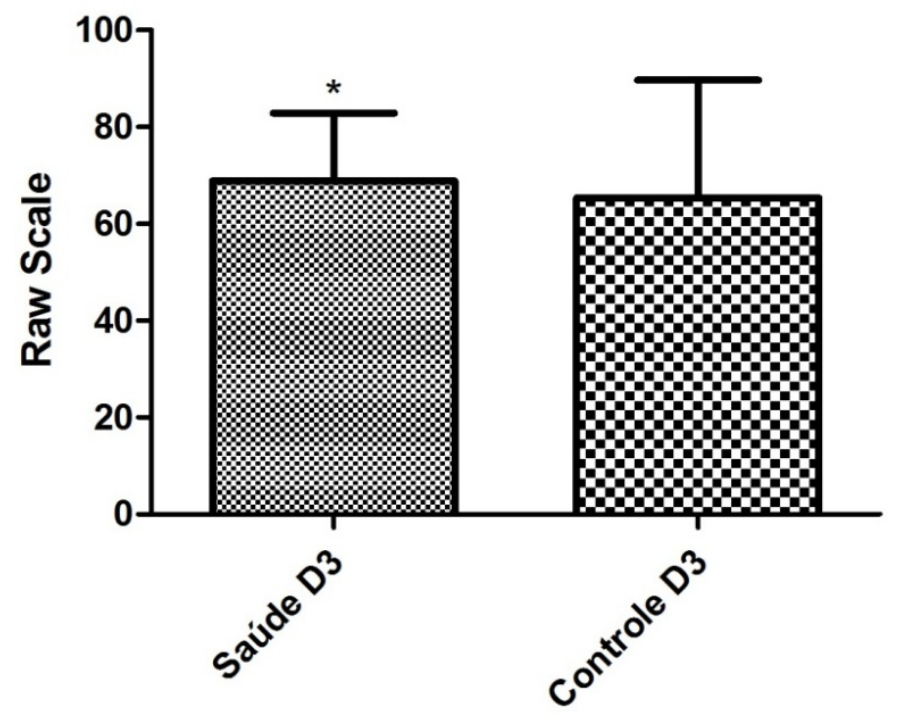

Fonte: dados coletados e tabulados pelos autores

Edição Especial com os melhores trabalhos apresentados no IV ENECiências: UFF - 13 a 16 de 
Figura 7: comparação do domínio 4 (estado geral de saúde - EGS) entre os grupos Saúde e Controle, por meio do teste $t$ de student não pareado $(p=0,0463)$.

\section{Estado geral de saúde (EGS)}

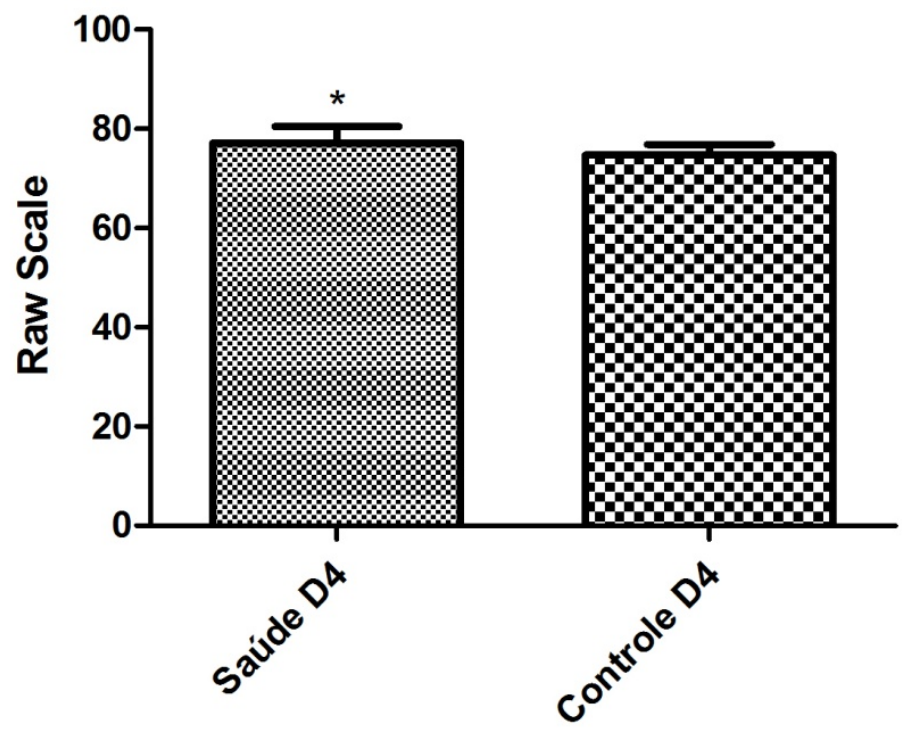

Fonte: dados coletados e tabulados pelos autores

Navega \& Oishi (2007) afirmam que o componente Estado geral de saúde considera a percepção do indivíduo quanto à sua saúde e o grau de concordância de quatro afirmações a respeito da perspectiva da sua saúde e a comparação em relação a outras pessoas.

Este grau de subjetividade condiz com a utilização deste instrumento em diferentes áreas da saúde para analisar a compreensão integral do indivíduo sobre sua saúde, no intuito de ajudá-lo a tomar decisões sobre seu tratamento, como exposto por Cesarino, et al. (2011), que também afirmam que nas últimas décadas as intervenções em saúde têm objetivado não somente o prolongamento da vida, e sim alcançar a melhoria de sua qualidade.

É possível aferirmos que os profissionais de saúde tendem a se autoconhecer de modo mais técnico, o que poderia ser traduzido em eficácia. Provavelmente a diferença encontrada entre os grupos Saúde e Controle nas dimensões "Dor" e "Estado geral de saúde - EGS" condiz com este princípio teórico. Porém, a semelhança encontrada nos demais domínios também aponta que profissionais de saúde docentes necessitariam dos mesmos cuidados em saúde que professores de outras áreas de atuação, independente de seu conhecimento técnico prévio em saúde. 
Assim, após visualizarmos os domínios obtidos por cada um dos cursos de graduação envolvidos, tanto no grupo Saúde, quanto no grupo Controle, cabe tecer o raw scale médio destes grupos, como demonstrado pela figura 8 abaixo, o que nos permite aferir que os domínios $3 ; 5 ; 7$, e exclusivamente para o grupo controle, o domínio 8, estariam abaixo de uma condição dita ideal de qualidade de vida; todos abaixo de $70 \%$ de eficiência.

Figura 8: Raw Scale médio para Os grupos Saúde e Controle.

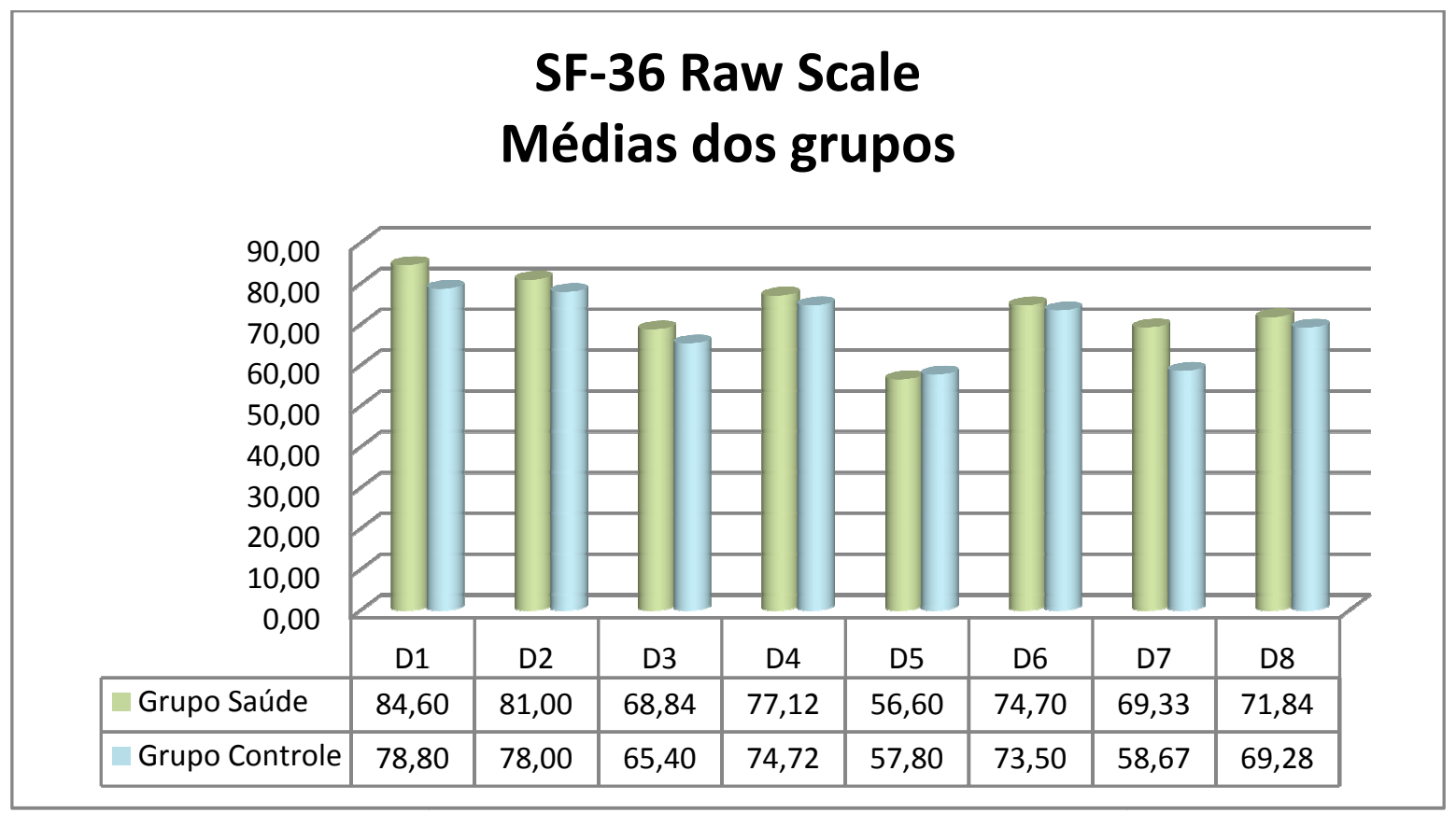

Fonte: dados coletados e tabulados pelos autores

Cabe ressaltar que nesta pesquisa não foi objetivada a análise da relação da carga horária trabalhada pelo docente com a sua qualidade de vida aferida, possibilitando futuras investigações a respeito.

\section{Conclusão}

Em nossa pesquisa foi possível verificar que existe um predomínio do gênero feminino preenchendo os cargos de professores em nível superior, sendo que os docentes da área de Saúde demoram mais para iniciar no Ensino Superior do que os professores formados por cursos de outras áreas, o que pode ser explicado pela formação de bacharel que estes docentes, profissionais de saúde, recebem ao longo de sua vida acadêmica. 
Dentre os indicadores de qualidade que foram estatisticamente semelhantes entre os dois grupos encontraram-se os domínios: 1 (Capacidade funcional), 2 (Limitação por aspectos físicos), 5 (Vitalidade), 6 (Aspectos sociais), 7 (Limitação por aspectos emocionais) e 8 (Saúde mental), o que sugere que os grupos de amostra e controle seriam comparáveis.

Estatisticamente observamos que apenas os domínios 3 (Dor) e 4 (Estado geral de saúde - EGS) apresentaram diferença entre os grupos, o que pode ser analisado perante o pressuposto de que os profissionais de saúde tendem a se autoconhecer de modo mais técnico, o que poderia ser traduzido em eficácia para sua qualidade de vida. Além desta diferença, também observamos uma correlação entre idade e tempo de atuação como professor no Ensino Superior.

Estas duas variáveis podem influenciar os resultados obtidos, principalmente no que tange o tempo de trabalho, já que outras pesquisas apontam que uma carga horária maior, ou um tempo de serviço mais prolongado pode influenciar negativamente a qualidade de vida de um trabalhador. Assim, pode-se compreender a naturalidade em se encontrar um maior índice de dor e um menor indicador de estado geral de saúde no grupo Controle, uma vez que este apresentou maior tempo de atividade profissional no Ensino Superior.

Entendemos também que outras pesquisas envolvendo a relação do tempo de atuação e a carga horária média trabalhada com a qualidade de vida dos docentes do Ensino Superior, independente da área de atuação, devem ser realizadas a fim de minimizar e / ou compreender as diferenças encontradas nesta pesquisa.

Em suma, a semelhança encontrada na maioria dos domínios aponta que profissionais de saúde docentes necessitariam dos mesmos cuidados em saúde que professores de outras áreas de atuação, independente de seu conhecimento técnico prévio em saúde. Para tal afirmamos que toda Instituição de Ensino Superior, ou outras instituições dos demais setores produtivos, devem investir na saúde de seu trabalhador por meio de programas institucionalizados de cuidados em saúde, desde a ergonomia e exercícios laborais até a recreação e socialização entre seus colaboradores.

\section{Referências}

BRASIL. Ministério da Saúde (MS). Conselho Nacional de Saúde. Resolução no 466 de 12 de dezembro de 2012. Diretrizes e Normas Regulamentadoras de Pesquisas Envolvendo Seres Humanos. Diário Oficial da União 13 de junho de 2013. 
CESARINO, C. et al. Qualidade de vida em pacientes com cardioversor desfibrilador implantável: utilização do questionário SF-36. Rev Bras Cir Cardiovasc, v. 26, n. 2, p. 238-43, 2011.

CICONELLI, R. M. et al. Tradução para a língua portuguesa e validação do questionário genérico de avaliação de qualidade de vida SF-36 (Brasil SF-36). Revista Brasileira de Reumatologia. v. 3, n. 39, p. 143-50, 1999.

FRAUCHES, C. C. (org). Diretrizes curriculares para os cursos de graduação. Brasília: ABMES, 2008. 702 p.

GALVÃO, L. L. L. F. et al. Prevalência de transtornos mentais comuns e avaliação da qualidade de vida no climatério. Rev. Assoc. Med. Bras., São Paulo, v. 53, n. 5, p. 41420, 2007.

GRINCENKOV, F. R. S. et al. Fatores associados à qualidade de vida de pacientes incidentes em diálise peritoneal no Brasil (BRAZPD). J. Bras. Nefrol, São Paulo, v. 33, n. 1, p. 38-44, 2011.

LAKATOS, E. M.; MARCONI, M. A. Técnicas de pesquisa. 7 ed. 5 reimpr. São Paulo: Atlas, 2011.

MOTA, L. M. H.; LAURINDO, I. M. M.; SANTOS NETO, L. L. Avaliação prospectiva da qualidade de vida em uma coorte de pacientes com artrite reumatoide inicial. Rev. Bras. Reumatol, São Paulo, v. 50, n. 3, p. 249-61, 2010.

NAVEGA, M. T.; OISHI, J. Comparação da qualidade de vida relacionada à saúde entre mulheres na pós-menopausa praticantes de atividade física com e sem osteoporose. Rev. Bras. Reumatol, São Paulo, v. 47, n. 4, p. 258-64, 2007.

SANTOS, F. R. et al. Efeitos da abordagem interdisciplinar na qualidade de vida e em parâmetros laboratoriais de pacientes com doença renal crônica. Rev. psiquiatr. Clín., São Paulo, v. 35, n. 3, p. 87-95, 2008.

SOARES, R. J. O. et al. Fatores facilitadores e impeditivos no cuidar de si para docentes de enfermagem. Revista Texto \& Contexto Enfermagem. Florianópolis, v. 20, n. 4, p.758-765, dez 2011.

SOUZA, J. C.; COSTA, D. S. Qualidade de vida de uma amostra de profissionais de educação física. J. bras. psiquiatr, Rio de Janeiro, v. 60, n. 1, p. 23-27, 2011.

TANI, G. Esporte, educação e qualidade de vida. In: MOREIRA, W. W.; SIMÕES, R. (org): Esporte como fator de qualidade de vida. Piracicaba: UNIMEP, 2002. 